\title{
The Use of Electronic Signature in Validation of Electronic Contracts from the Jordanian Perspective
}

\author{
Enas M. Qutieshat \\ School of law, Philadelphia University, PO box 11, Amman 19392 Jordan
}

\begin{abstract}
It is common these days to form contracts electronically. The verification of the electronic signatures is an important condition, which needs to be fulfilled according to the Jordanian laws. The reason for this is that, according to Article (30) of the Electronic Transaction Law, electronic signatures are considered to be evidence that the contracting parties have legally consented to the contents that are contained in a document. This article will highlight the current approach on the subject matter from the perspective of the Jordanian law and if there are any Limitations concerning the E-Signatures in the Jordanian Law.
\end{abstract}

Keywords: electronic signature, electronic contracts, validation ,authentication

DOI: $10.7176 / \mathrm{JLPG} / 89-09$

Publication date:September $30^{\text {th }} 2019$

\section{Introduction}

Effective business transactions are reliant on two components - speed and security (Zhu, 2013). Successful businesses in the 21st century are the ones that can acquire information quickly, adapt to the changes that are taking place in the business environment (Shahjee,2015). The advancement of technology and assimilation of business transactions to modern technology have ensured that a majority of the businesses transactions today do not rely on contracting parties meeting physically. Instead, business transactions can be completed electronically, which ensures that they speed up the process, and increase the level of trade efficiency. In the 21 st century, trading is not only confined to national boundaries mainly because of globalization, and information integration, and therefore, there is the need to exercise caution when it comes to finalizing transactions and contracts electronically, or through the use of the internet, especially when dealing with businessmen from other countries. The reason for this is that when using the digital platform, businessmen are exposed to risks such as violation of privacy, cyber-crime, and ambiguity in the set laws for dealing with online transactions. One of the accepted ways of securing online transactions is through the use of an electronic signature (Al-Zubi \& Al-Trawneh, 2016).

From 1995, Jordan has been taking the right and necessary steps in its development of the networking and technological parameters to facilitate the electronic commerce sector in the country. The e-signature is one of the most important elements that is needed to facilitate a successful electronic commerce sector (Shnikat, Alzubi, Aljaber, \& Alnsoor, 2017). However, although, Jordan as a country has been keen to implement legal policies in regards to the e-signature, there is still a major issue in terms of the following parameters of the identification, and authentication of the e-signature during the finalization process of a contract. The Electronic Transaction Law (ETL) of Jordan currently provides two ways of verifying e-signatures, and this creates a sense of ambiguity at times during the interpretation of the law.

\subsection{Problem Statement}

The electronic signature was designed to perform similar function as the traditional signature whereby, it acts as proof that the signatory is satisfied with the contents that are contained in a document. However, the two ways that are provided by the ETL of Jordan for verification purposes (objectivity, and formal conditions) have resulted in ambiguity during the interpretation of the law. In addition to that, there are various aspects of the esignature that require to be elaborated as they are not contained in the ETL such as a clear definition of the esignature, validity process, and recognition of the foreign certificates. The subject matter of this paper is important since that the Jordanian Evidence law was modified in 2017 by adding sub-paragraph 3 of article 13 of law. Previously the law only considered letters sent via email to be considered as regular papers in proof and evidence without setting any conditions or referring to the Electronic Transaction Act, but after the modifications were made as the law required in some cases a testimony from the sender to assure that he is the one who sent the email, and in other cases, the law accepted the use of the email as a mean of proof without the need of having a testimony. .

\subsection{Purpose of the Study}

The purpose of this study is divided into two sections. Firstly, the researcher will identify the functions of the esignature as defined by the ETL of Jordan. Secondly, the researcher will evaluate the issues of the e-signature, and how they can be corrected, or adjusted through making amendments of Jordan's electronic transaction law. 


\subsection{Importance of the Study}

E-commerce has become a global phenomenon in that, across the world, technological advances in the ICT field has resulted in a majority of businesses using the internet to sell their goods, and offer services to their clients. In 1995, the total global e-commerce was zero, and by 2018 , it was estimated that the total e-commerce users globally were 1.79 billion, which represented $22 \%$ of the business owners worldwide, transacting approximately USD 2 trillion via the internet (Statista, 2019). Today, a majority of businesses have an online presence whereby they are able to increase and diversify their business' customer base from only dealing with the traditional faceto-face consumers to transacting with clients using the internet platform. Such statistics are an indication of the growth and potential of online businesses. With that stated, there is the need to implement laws that ensure the online contracting parties are protected from unscrupulous activities.

\subsection{Objectives of the Study}

This research paper aims to tackle the following research questions:

1.What are the functions of the electronic signature as defined by Jordanian's electronic transaction laws, and their purpose in the validation of electronic contracts?

2.What are the shortcomings of the electronic transaction law in relation to the electronic signature in Jordan?

\subsection{Research Methodology}

In this research paper, the researcher adopted a purely legal research methodology. As this is a legal research, the researcher applied the interpretive doctrinal technique, which is commonly known as the library research. The data that was collected was from the Electronic Transactions Law No. (85) of 2001, and UNCITRAL Model Law on Electronic Signature with Guide to Enactment 2001. The data was analyzed using a comparative approach to assess the use of electronic signature in the validation process of electronic contracts from the Jordanian perspective. The researcher was interested in determining the functions, and the legal conditions of the e-signatures using the electronic transaction laws of Jordan.

\subsection{Structure of The Study}

This research paper is divided into four parts. The first part is the introduction to the work, it identifies the problem of the study, the purpose and importance of the study in addition to the objectives and research methodology. The second part is literature review section on electronic signature that gives a brief review on this type of signature. This part contains the definition of an electronic signature. It also has a section of the advantages of an electronic signature, capacity needed to enter into an electronic contract, legal challenges encountered when concluding an electronic contract, and court cases involving the use of e-signatures in an electronic contract. The third part is the legal conditions of the e-signatures in accordance with the Jordanian ETL whereby the researcher provides evidence that e-signatures are considered to be proof of contracts conclusion in electronic contracts according to the Jordanian law, and the legal conditions of the e-signatures in accordance with the ETL. The fourth part is the functions and authentication of the e-signatures. In this section, the researcher provided a list of the functions, and the authentication process of the e-signatures. Lastly, the conclusion and recommendations section provides a brief overview of the research paper, and the approaches that can be undertaken by the Jordanian legislators to improve the current ETL to ensure that electronic signatures can be used in the validation of electronic contracts in the country.

\section{Electronic signature: a brief review}

A signature, in general, is considered as a sign, or symbol that acknowledges satisfaction of proof of the contents that are contained in a document, and that sign, or symbol is only attributed to a particular person who is considered as its only user (Government of Jordan, 2001). According to Article (2) of the Electronic Transaction Law (ETL) of Jordan, the electronic signature is described as:

"Electronic, numeric or photo data or others taking the shape of letters, numbers, symbols, or signs, or the like in a data message or added or related thereto, having a shape identifying the person who timed or distinguished it from others for reasons of the person's signature and the approval of the content (Government of Jordan, 2001)."

Almaharmeh (2016) notes that according to the Jordanian law, the purpose of the electronic signature is the same as that of a traditional signature. For instance, a traditional signature can be used as evidence, for ceremonial function purposes, as a designation of approval, and proof of authenticity. An electronic signature can fulfil the same functions as those of a traditional signature in an equivalent manner.

What becomes clear is that the definition of the electronic signature is ambiguous in that it fails to explain the issuance, and process of authentication of the signature. However, according to the country's legislation in Article (10/b) it highlights the validation process, and functions of the e-signature as follows:

"The validity of the signature shall be proven and attributed to the person signing the electronic record 
when there is a method to identify that person and to indicate his approval of the information contained in the electronic record that carries his signature, if that method is reliable for this purpose in light of the circumstances relating to the transaction, including the parties' agreement to using this method (Government of Jordan, 2001)."

Shnikat, Alzubi, Aljaber and Alnsoor (2017) note that although both the traditional, and electronic signatures are used for the identification of the signatory, and as proof of an individual's satisfaction of the contents that are contained in a document, there are differences between these two types of signatures. The traditional signatures are considered to have a high level of privacy, confidentiality, and safety, which is not the case for the electronic signature (Shnikat, Alzubi, Aljaber \&Alnsoor, 2017).

\subsection{Advantages, or Benefits of an Electronic Signature}

Almaharmeh (2016) stated that the electronic signature was developed as an independent system to assist in boosting the confidence and security of online transactions, and to ease the verification of the signatory parties of a contract. It is important to point out that both the e-signature and the traditional signature have numerous similarities in terms of functionality, and as the e-signature began to become more popular, the use of the traditional signature ultimately began to decline (Almaharmeh, 2016). The reason for the increase in the usage of the electronic signature is that it exceeds a majority of the challenges that have been associated with the traditional signature such as dealing with paper documents that can easily be lost, and its limited space for archiving purposes. In addition to that Shnikat et al. (2017) noted that, the electronic signature has more varied features than the traditional signature concerning privacy, integrity, reliability, and lack of denial (Shnikat, Alzubi, Aljaber, \& Alnsoor, 2017).

According to the Government of Jordan (2001), the use of the electronic signature allows for the integration of the documents that are used in a contract. This means that the documents cannot be changed, or modified once they are dispatched by the sender to the receiver. Also, it is easy to prove that there was tampering of the contents when the documents are sent electronically, which will make the e-signature to be null and void (Government of Jordan, 2001). It limits the ability of fraud taking place during the process of signing the contracts.

Almaharmeh (2016) stated that the use of electronic documents, and e-signatures during the finalization process of a contract means that they are inadmissible to other parties. It limits the people who have the right to see the documents' contents to be the sender, and the receiver as they are the only ones who possess the public, and private key (Almaharmeh, 2016). Although one can make the argument that there is also privacy within the traditional signatures framework, they do not have this level of degree of privacy as can be achieved when using the e-signatures.

Almaharmeh (2016) added that the use of e-signatures ensures that the sender of the documents is aware of the arrival of the electronic documents to the receiver. This is particularly essential when dealing with cases whereby in the past, a receiver could have denied ever receiving documents for an intended purpose, and therefore, absolved themselves from responsibility, or liability that has been stipulated by law (Almaharmeh, 2016). When using electronic documents that have an e-signature, the sender not only knows that the electronic documents have arrived, but, they also have the time stamp, and evidence of this action, and therefore, the receiver cannot legitimately deny that he or she has had access to the documents.

According to Shnikat et al. (2017), another benefit of the e-signature is that its date cannot be modified or deleted, and therefore it enhances its validity. This is especially essential in situations where fraudulent events are highly likely to happen (Shnikat, Alzubi, Aljaber, \& Alnsoor, 2017). Electronic documents that have been signed using an e-signature will have a time stamp, and therefore, there is no need to speculate on when the document was signed, which can affect the overall eligibility of a document.

Almaharmeh (2016) stated that the use of electronic signatures ensures that the contracting parties are in a position where they can verify the source of the message and its sender. It can, therefore, be used as part of the authentication process, and also to verify the identity of the public keys before the completion of a contract. On the other hand, although the traditional signature is considered to be unique, and can only be identified with a certain individual, for this to be completed, it has to be scrutinized, and analyzed by all the contracting parties before it can be accepted (Almaharmeh, 2016).

Electronic signature come in varouse types for example :

\section{Signing by Ending with the Sender's Name}

In this e-signature, it is completed by printing the name, and title of the sender on the electronic document. The printing of the name, and address at the end of each document is conducted in an electronic format using an electronic mail (e-mail), Word (WORD document), Excel, and the HTML.

\section{Electronic Signature with a Pen (PEN-Op)}

This e-signature is inscribed on an electronic document using an electronic pen, which can write on a computer screen, using a specific program. The program can fulfill two functions. Firstly, it can capture the signature. 
Secondly, it verifies the signature as the program can receive the customer's data through the placement of a card on the machine, and then a message will appear.

\section{Scanning Signature}

When using a scanner, the signature is transferred to a file where it was intended in the form of an image. The image is developed from a traditional signature that is scanned, and transferred to an electronic document.

\section{Biometric Signature}

When using this type of e-signature, it is dependent on the physical, and chemical properties of the individuals involved such as the use of personal fingerprints, human eye scan, level and tone of the voice, and the properties of the human hand for the identification of an individual's human face, and personal signature.

\section{Click Signature}

This type of signature works similarly as is the case when for instance, an individual is clicking 'OK,' or 'I accept,' in a document. This type of signature is used during purchasing and also when conducting, or finalizing business transactions for approval purposes. This type of signature is usually linked to what is known as (clickwrap agreements)..

\section{Pin-Code}

It is one of the most common e-signatures that is used globally. It is used using magnetic strips, or small electronics that are in-built in cards to ensure that the transactions are secure, for instance, a bank account card that people use in the ATMs to make withdrawals

\section{Digital Signature}

When using this type of signature, the data system exists in the form of a code, which ensures that the all the parties involved in a contract are aware of the origin of the e-document, and its contents. Examples of digital signatures are coded public, and private keys

\subsection{Increases the Speed and Accuracy During the Conclusion of a Transaction}

When using the traditional signature, the contract council meets with the contracting parties to complete the signing process of a document. Before its finalization process, it has to be legally authenticated, and expression of consent of the contents of the documents by all the parties that are involved (Almaharmeh, 2016). However, when using the e-signature, the contract council does not have to necessarily meet with the contracting parties to conduct the processes that have been mentioned in the previous sentence. In addition to that, the contracting parties do not have to be physically present to one another to witness the signing process, instead, it can be completed using e-mails through the electronic authentication. It speeds up the process and ensures that it is secure (Shnikat, Alzubi, Aljaber, \& Alnsoor, 2017).

\subsection{Capacity to Enter into an Electronic Contract}

Mormann (2015) stated that the eligibility of an electronic contract that is remotely contracted is difficult because of the issue of one of the contracting parties verifying the eligibility of the other contracting party. The geographic, and spatial separation of the parties that are involved in the electronic transactions can result in lack of knowledge of basic information about each other. Hanadi (2015) states that to legally conclude an electronic contract, the parties entering into a contract should be verified. Whitley, Gal and Kjaergaard (2014) noted that the contracting parties have to check and confirm the identities of the other contracting parties. Kalloniatis et al. (2014) agreed that the contracting parties should be obliged to provide identification data as is the case in other contracts.

\subsection{Legal Challenges during the Conclusion of an Electronic Contract}

The most common way of conclusion, or completion of an electronic contract is by communicating through electronic correspondence such as the e-mail. In general, one side of the contracting parties will provide the conditions, and if there is an agreement by the other party, then, the agreement will be concluded. However, there is a possibility of the following problems arising. Mukherjee and Nath (2003) stated that confidentiality, and privacy are considered to be challenges that affect the acceptance of some customers to the idea of online shopping. For instance, during an electronic exchange process, there is data that is needed from the customers such as name, gender, nationality, and method of payment. Ghosh, Mahdian, McAfee, and Vassilvitskii (2015) state that there is the need to use special software to maintain the confidentiality, and privacy of the exchanged data during an electronic transaction.

McKendrick (2014) stated that the parties of an electronic contract need to know the due date for the conclusion of a contract. The reason for this is that when a contract is composed, the parties that are involved become legally associated and have obligations that they have to fulfil. Also, the time of formation of a contract may have a critical impact in reporting certain issues that pertain to the contract such as right to withdraw, price of the products, and time of transfer of ownership of products (Ramokanate, 2014).

According to Chan (2014), the condition of a signature can be an authentic electronic contract, or 
mandatory legal power of the contracting means. Bagherian, Nasaband, Heidari and Ebrahimi (2014) stated that in a traditional trade, the signature of a person on a written contract is a representation of proof of authentication. In unwritten contracts, the evidence from the witness is important is enforcement of the obligation parties after the conclusion of a contract (Ramokanate, 2014). McKendrick (2014) observed that the electronic signature in most cases where it is used is able to achieve the function of a traditional, or physical signature.

\subsection{Court Cases on the Use of E-Signatures}

At the time of writing there is no law case brought to the Jordanian law regarding e- signature, nevertheless there are many cases worldwide. For example in Barwick v. GEICO, through the use of a click-to-sign e-signature, an insurance client waived the minimum medical coverage on her insurance plan. Later on, after suffering an accident, which led her to incur medical expenses, the insured claimed that the electronic signature was not binding because it was not in 'writing.' The lawyer of the insured party claimed that a general statute such as the UETA cannot be applied when a specific statute such as an insurance statute stipulates that all contracts have to be 'in writing.' The Arkanas Supreme Court made a ruling whereby it stated that there was no conflict between UETA and the auto insurance statute. It noted that UETA was straightforward in allowing an electronic record to satisfy the law that requires a record to be in writing. In this case e-signatures are considered to validate electronic contracts (Lexology, 2011).

In $O^{\prime}$ Connor v Uber the plaintiff argued that the electronic contract was not a valid agreement because it was displayed 'on a tiny' iPhone screen when a majority of drivers are about to start their shift. However, the court rejected this argument. It ruled that it is irrelevant whether the other contracting party reads the contract as long as they are provided with an opportunity to do it (Justia, 2018).

In another case, in the famous case of Zakuski v General Americana doctor took out a \$250,000 life insurance policy, and he named his mother as a beneficiary. However, he married a second wife, and it is claimed that he changed the beneficiary designation to her. General American allows its customers to make changes of the beneficiaries of its life insurance policy online as long as they provide information on the policy number, Social Security number, and the maiden name of the beneficiary. The company then sends an e-mail for confirmation purposes. The evidence provided was that someone accessed the General American e-service as the doctor and provided the required information, and changed the beneficiary to the second wife, and an e-mail alert was sent. The doctor died shortly after these changes were made, and the mother sued the insurance company claiming that the company's security policies were not enough to warrant the changes, and that General American could not prove that it was the doctor who made the changes, and not the second wife (Assuresign, 2018). The Michigan court upheld the e-signature stating that the required information presented by General American was provided, and an e-mail alert was immediately sent. In addition to that, the court observed that the doctor's mother did not provide any evidence that someone else other than the doctor had made these changes. In this case the General American procedure of validating the information presented online by a contracting party including the e-signature was considered sufficient.

\section{Legal Conditions of the E-Signature According to Current Jordanian ETL}

\subsection{E-Signatures as Proof Contracts Were Concluded Through the Internet}

Even though the ETL is considered the main Jordanian legislation that is used in the determination, and provision of governing parameters for the e-signatures, other legislations can be used in this process such as UNCITRAL 2001, the ECD 1999, and the E-SIGN. The purpose of this legislation is to assist in the process of legalization of all the aspects that are required during the e-signatures process.

The implementation of the e-signatures in the execution of legal binding processes will be evaluated in three stages. Firstly, it is important to assess the situational parameters of e-signature (Bain \& Subirana, 2003). Secondly, one needs to determine the duties and responsibilities of the different parties that are involved in an electronic contract. Lastly, there is the need to assess the impact of the foreign signatures if they are part of the contracting parties. The reason for this that currently the foreigners' e-signatures are not covered by the ETL in Jordanian legislation (Government of Jordan, 2001).

\subsection{The Legal Conditions of E-Signatures}

The validity of an e-signature relies on its fulfillment of the set functions that are to be performed by the signature, and determination of the parameters under which the signature is provided. However, there is a shortcoming in the Jordanian legislation in terms of covering the aspects that have been mentioned. Generally, the e-signatures have the same legal weight as the traditional written signatures, if they are both signed in a manner, and in conditions that fulfill the standard parameters of a signature in a legal document.

The legal standing of the e-signatures is governed using two tiers of legalities. If the standard functions of a written signature are performed using an e-signature, then it is considered as a 'general validity in proof.' Secondly, as has been stated before, an e-signature is determined as valid if it can withstand scrutiny in a court of 
law without the need of the traditional written signature as determined by Article (5/1):

"The Member States shall ensure that advanced electronic signatures which are based on a qualified certificate and which are created by a secure-signature device (a) satisfy the legal requirements of a signature in relation to data in electronic form in the same manner as a handwritten signature satisfies those requirements in relation to paper-based data, and (b) are admissible as evidence in legal proceedings" (Government of Jordan, 2001).

However, there is a need for specific technical parameters to be addressed before this high level of practicality can be realized. It has been determined that while there are set of laws that are set in broad parameters to equate e-signatures with the traditional written version, there are others that focus on different parameters such as integrity, security, and confidentiality (Ihab, Ismail, \& Kamat, 2006).

If a signatory desire that an e-signature be considered from a legal perspective as the traditional written signature, the following considerations need to be fulfilled. The signature that a signatory is willing to place on a legal document should be uniquely associated with him, or her. The characteristics that are contained in the esignature should be able to identify the identity of the signatory (Ihab, Ismail, \& Kamat, 2006). The signature should be used in a manner that just like the written signature, it cannot be altered by unauthorized third parties. It is also important to point out that the e-signature should be linked to an entire document through a particular page in such a manner that it cannot tamper, or altered to affect the originality of the page and document. If there is any form of alteration, it should be easy to detect to ensure that further action is done (Ihab, Ismail, \& Kamat, 2006).

If e-signatures can fulfill all the stipulations that have been mentioned, and described in the above paragraph, then without a doubt, it can, and should have the same legal weight as the traditional written signature. In addition to that, it can even be considered as an advanced, or enhanced signature. However, taking into consideration the e-signatures and the Jordanian laws that are set to govern, and regulate them, one notices that there are certain existing anomalies, which are listed in the next section (Ihab, Ismail, \& Kamat, 2006).

\subsection{Limitations in the Jordanian Law concerning the E-Signatures}

Article (2) of the ETL provides a general definition, and overview of the e-signatures. However, it does not provide the specifics that can be used for its authentication process, which can be considered as a loop-hole in the law (Government of Jordan, 2001). Article (10) of the ETL only provides the general parameters of the validity and basic functions of the e-signatures. Article $(32 / b)$ has been described as 'the confusing rule.' The reason for this is that, in this section of the law, there is the stipulation that both the authentication process and parameters must be fulfilled for the e-signatures to be considered valid over a long duration (Government of Jordan, 2001).

According to the aspects that have been mentioned in the previous paragraph, for e-signatures to be considered valid in the Jordanian law, they need to be authenticated. However, there is the need to take into consideration other parameters such as technological advancement whereby the contracting parties can use encryption to ensure that the documents or certificates used in a contract cannot be accessed by unauthorized parties (Ihab, Ismail, \& Kamat, 2006). This type of technology (encryption) can be used for authentication purposes. Therefore, there is the need to amend the Jordanian legislation, in particular, the ETL, to ensure that there is no existing conflict between the different aspects of the law that are related, or can be used to address issues in the e-signature incidences. All the laws that are enacted, which are related to the e-signatures should be in perfect synchronization to each other. They should also complement, and supplement each other to avoid ambiguity in the Jordanian legislation. Moringiello and Reynolds (2005) pointed out that the issue of the legality of the e-signatures has been dealt with in the judicial system. For instance, in the US judicial system, the aspect was addressed as the functions and conditions of the e-signatures have been defined appropriately (Moringiello \& Reynolds, 2005).

\subsection{Liabilities that Result from the Use of the E-Signatures}

The ETL fails to address the issues of obligation, and requirements for the contracting parties that are entering into an agreement using an e-signature. However, the UNCITRAL 2001, and ECD 99/93 provide obligations to the parties (signatory, relying party, and certification provider) that are using e-signatures to finalize their contracts.

\subsubsection{Signatory Obligations}

Article (8/1) of the UNCITRAL 2001 places the following responsibilities to the signatory of an e-signature. Firstly, the signatory needs to exercise reasonable care when using the e-signature to ensure that unauthorized parties do not have access to the signature creation data (United Nations, 2001). Secondly, the signatory should utilize any of the means available that have been provided by the certification service provider during the pursuant of article 9 of the Law. In addition to that, the signatory should notify the other contracting parties and certification service provider if they are aware or suspect that the signature creation data has been compromised, 
or the circumstances that have been made available for the signatory possess substantial risk, and the signature creation data may have been compromised (United Nations, 2001). Also, in situations whereby a certificate is used to support the validity of the electronic signature, there is the need to ensure that the materials that are represented by the signatory and certificate are relevant throughout the lifecycle of the e-signature. Article (8/2) of the UNCITRAL 2001 states that; "A signatory shall bear the legal consequences of its failure to satisfy the requirements of paragraph 1" (United Nations, 2001).

\subsubsection{Certificate Service Provider Obligations}

The duties and obligations of a certification service provider are stipulated in Article (9) ${ }^{18}$ of UNCITRAL 2001 (United Nations, 2001). If the certificate service provider fails to execute any of the duties, or obligations that have been accorded to him, then he is considered to be legally liable for any discrepancies that will be detected in the process. It is important to point out that in Article (6) ${ }^{19}$ of the ECD 99/93 there are unambiguous directives that are accorded to the certification service provider to ensure that both the level, and standard of certification provided can stand up to not only public scrutiny, but also any public evaluations, and analysis that the certification process will be subjected to, at any given moment (United Nations, 2001).

\subsubsection{Relying Party}

The conducts that are accorded to the relying party are stipulated under UNCITRAL 2001, Article (11). In this section of the law, it clearly states that the relying party in the event of a failure on his, or her part shall have to bear legal consequences. A relying party also must verify the reliability of an electronic signature, and even when the e-signature is supported by a certificate to ensure that the certificate is valid, and any form of limitation that is related to the certificate. It is important to point out that the legalities of this aspect have been covered under Article (33) of the ETL (United Nations, 2001). However, although it has been covered under the Jordanian Law, it is considered to be too generalized giving room to multiple interpretations. It should be more specialized and detailed to ensure that it can stand up to any form legal scrutiny, analysis and review. Therefore, the Jordanian law should be revised in a way to address the gaps and loopholes that have been identified, especially in the ETL to address the issues that have been identified with the e-signatures, especially in agreements, and finalization of contracts so that they can stand up to legal scrutiny, and evaluation (United Nations, 2001).

\section{Functions and Authentication of the E-Signatures According to the Jordanian Law 4.1 Functions of the E-Signatures}

The functions of the e-signatures are identified as follows:

- It is used to identify an individual who is related to a specific electronic signature. In addition to that, the contracting parties can use other methods for identification purposes such as the Digital ID, and personal certificate, which can be sent via the internet. All these methods are used for verification purposes for the individuals who are involved in a specific contracting process.

- The signing of a document using the electronic signature is considered to be proof that a person or one side of the contracting party has agreed to conclude the contract. In addition to that, when there is an electronic signature in a document, it is an indicator that an individual agrees with the legal framework that is contained in the contract, and he or she is ready to fulfill the set obligations that have been stipulated in that contract.

- The e-signatures ascribe to the objective and formal conditions that were set in the Jordanian legislature.

\subsection{Objectivity Conditions}

According to the Jordanian laws, for an e-signature to be considered valid, it must fulfill the verification process, and conditions of the e-signature, which are discussed in this section.

\subsubsection{Authentication}

The verification of the e-signatures is an important condition, which needs to be fulfilled according to the Jordanian laws. The reason for this is that, according to Article (30) of the ETL, e-signatures are considered to be evidence that the contracting parties have legally consented to the contents that are contained in a document. This is achieved using the following conditions. Firstly, the verification process that will be used should be an approved process. The approval is normally conducted by a governmental or non-governmental agency that has been provided with the mandate of verification, and it is usually achieved through the use of e-signatures and erecords. It is conducted to ensure that the legality of the process is affirmed.

The second condition is commercial acceptance, which is specified in Article (30/b) of the ETL, and the contracting parties should take into consideration the commercial aspects of the e-signature. The third condition is that the agreement between the contracting parties should contain all the processes that these parties think, or assume will apply in specific transactions. It should be framed in such a way that it benefits all the parties involved in the fulfillment of a specific transaction (Government of Jordan, 2001). 


\subsubsection{Conditions Needed in the Authentication Process of an E-Signature}

Besides the verification of an e-signature, there are other requirements by the Jordanian laws that are needed to ensure that it meets, or fulfills all the considerations for legal significance in terms of consenting by the involved parties. Article (31) of the ETL contains these necessary conditions (Government of Jordan, 2001). A valid esignature should fulfill all of the following characteristics. Firstly, it should be one of a kind, or unique, and related to the correct person. This means that even when there are multiple e-signatures on different legal documents, it should be easy to differentiate one signature from another. Secondly, the e-signature on its own should be enough for the identification of a person who produced it. This is tied to the first characteristic that stipulates e-signatures are supposed to be unique (Shnikat, Alzubi, Aljaber, \& Alnsoor, 2017).

Thirdly, the e-signatures should only be produced and regulated by their rightful owner. In addition to that, they should only be used by the owner or an individual that has been authorized by the owner of the e-signature. It means that the owner is the only one who has exclusive rights for the usage of the e-signature, and there is no way that it can be produced, and used by unauthorized parties. Fourthly, the e-signatures should be linked to the e-records directly. The connection between the e-signatures and e-records should at no time be compromised by unauthorized parties (Al-Zubi \& Al-Trawneh, 2016). The reason for this is that if any other person, or party that has not been authorized to access the signatures, or records get access to them, it means that they can use the esignature without the consent of the rightful owner. If this happens, the e-signatures will lose their validity as its owners will not possess the control of their usage.

\subsection{Formal Conditions}

Jordanian regulations that are concerned with specific parameters such as authentication, validity, and identification symbols are contained in the ETL and will be discussed in this section.

\subsubsection{E-Signatures are Supposed to Be Signed within a Specific Validity Period}

All legal documents and certificates that are issued during the duration of a contract have a specific validity period, which, after expiry, will bear no legal clout. Similarly, e-signatures are required to be placed on a document within a specific authentication period to ensure that a document or certificate will contain the required legal weight. If the signature is placed after the expiry of the period, it will fail to fulfill the objectivity parameters of the e-signature concerning authentication.

\subsubsection{The Authentication Certificate Needs to Correspond with the Identification Symbol}

The authentication certificate should correspond with the authentication symbol. The reason for this is that it ensures that only the concerned, and related parties will have access to the contents that are contained in an electronic file. If this is achieved, then the privacy of the contents of the private file is maintained as indicated in Article (34) of the ETL (Government of Jordan, 2001). Technological advancement has necessitated the use of esignatures in the finalization of agreements and contracts. As has been shown before, there is the need for maintaining and ensuring that the electronic records cannot be accessed by unauthorized parties and that there are legal frameworks, and regulations that are contained in a country's law for authentication of the e-signatures. However, even though the ETL addresses a majority of the legal aspects that are related to the e-signatures, there are situations whereby there is the creation of problems in terms of interpretation of the law (Al-Zubi \& AlTrawneh, 2016). For instance, there are discrepancies in Article (2) of the ETL (Government of Jordan, 2001). In this section of the law, even though it has specified the situations whereby e-signatures are applicable, it fails to provide the ideal verification requirements.

In the ETL of the Jordanian legislation, two parameters have been set for recognition, and validity of the esignatures and they need to be fulfilled to ensure that a certificate can stand the scrutiny process in a court of law. The parameters that have been mentioned are contained in Articles, 7, 10,30,31, and 32b of the ETL (Government of Jordan, 2001). An important point to note is that, according to these sections of the law, the esignatures should be authenticated at all times to ensure that they can withstand legal scrutiny. However, there is no section, or sub-section in the ETL that provides explanations, and directives on how the authentication process needs to be coordinated.

\subsection{Recognition of Foreign Signature and Certificate}

The legalities of the foreign e-signatures are contained in Article (12) of UNCITRAL, Article (34) of the ETL, and Article (7/1) ${ }^{23}$ of the ECD 99/93. However, the Jordanian legislation should be revised to ensure that the existing laws such as the ETL can fully govern, and regulate the usage of foreign e-signatures. The Jordanian regulations, for instance, should address specific signatures as has been listed in UNCITRAL 2001 to ensure that international contracts that have been finalized using the e-signatures can withstand legal scrutiny if there is any need (United Nations, 2001). This is to eliminate ambiguity or any loopholes that currently exist in the Jordanian legislation when dealing with foreign e-signatures as has been observed in the local, or national e-signatures. 


\section{5 electronic signature in the Jordanian Evidence law number 30 of 1952.}

The reader of this law can find that the legislator took in account that in some cases courts will be faced with cases that include the use of emails in the process of communicating between parties to reacg agreement. Therefore the article 13 of the evidence law before modification stated that 'Fax, telex and electronic mail shall have the force of regular papers in evidence.' Later on and by law number 22 of 2017 this article was modified to read as follows :

"3. (a) Subject to this sub-paragraph, fax, telex, e-mail, and similar means of modern communication shall have the power of regular papers in proof, if accompanied by a testimony from the person who sent them to confirm their issuance thereof, or by a testimony of receipt to support the receipt thereof unless otherwise proved.

(b) E-mail messages shall have the power of regular papers in evidence without being associated with a testimony if the conditions required by the Electronic Transaction Act are met.

(c) It may be agreed that the data transferred or preserved using modern technology through a secret number agreed between the parties shall be an used against each of them to prove the transactions made under the use such data.'

The reader of this article finds that the legislator took in account that an email could be signed traditionally by inserting the name of the sender at the bottom of the email or digitally . in the first case the law required testimony from the person who sent them to confirm sending the email, while in the later case the law refereed to the ETL. Having said that , both laws still have left out electronic contracts which were made by a click of mouse or what is known as browse-wrap agreement. There for the click of mouse is still in question in Jordan as whether it could be a valid method of signature or not.

\section{Conclusion and Recommendations}

As has been indicated by the researcher in this research paper, there are various aspects of the e-signature that require to be elaborated and clarified as they are not contained in the ETL. These issues include clear definitions of the e-signatures, its validity, authentication process, and the recognition of the foreign certificates. Although the Jordanian legislation through the ETL has defined e-signatures in Article (2), there are shortcomings such as determining the authenticity, and genuineness of the e-signatures. In particular, in Article (2) of the ETL, the authentication process has only been partly defined. Another major issue that the researcher observed is the identification and authentication of the e-signature. There is the need for the laws to be properly amended to ensure that the way the e-signatures are interpreted in a court of law perspective is the same as that of the traditional signatures. This will be crucial in ensuring that the electronic signatures are used in the validation of the electronic contracts in Jordan successfully.

\subsection{Recommendations}

- There is the need to have a clear definition of the e-signature as is the case of the traditional signature to ensure that they fulfil the same function.

- Currently, in the ETL, two ways can be used to verify the e-signatures, and this leads to a sense of ambiguity. The researcher proposes that there are need to either do away with one of the approaches, or synchronize Articles (7,10 and 30), and Articles (31, and 32/b).

- The guidelines and regulations provided in these Articles are supposed to complement each other, as opposed to creating ambiguities as is the present state, which can create legal loopholes and reduce the functionality level of the e-signatures.

- The ETL should provide clear frameworks on the legal procedures that needed to be followed when dealing with international contracts. This can be achieved through cooperation between countries that Jordan trades with online to develop uniform and specific laws that deal with electronic contracts.

- There is the need for collaboration between the legal and IT professionals to come up with more efficient electronic laws that will reduce the loopholes that can be used by unscrupulous businessmen during the formation, and conclusion of electronic contracts.

- There is a need to cover other types of electronic signatures by the Jordanian legislator specially those that are linked to a document by a mouse click since it is commonly these days that users will not read the long list of terms that are associated with the (I accept) bottom.

Finally, based on this article further future work could take place to analyse the Jordanian customer behaviour regarding the use of electronic signature in their daily work and the impact that such use might have on their legal state. Further work could also be carried as to study and analyse the possible point of view of the Jordanian courts regarding cases were the case involve the use of electronic signature this could be done in an analytic approach by questioning judges and other role players that are involved such cases.

\section{References}

Alalo, H., Wishah, R., Alali, S., Al-Sukkar, A., \& Abu-Hussien, A. (2016). E-marketplace legal and regulatory 
framework in Jordan: A general view. Journal of Theoretical and Applied Information Technology, 85(2).

Almaharmeh, M. M. (2016). Authenticity of Electronic Signature Under Jordanian Legislation. Journal of Law, Policy and Globalization, 56.

Al-Zubi, A., \& Al-Trawneh, B. (2016). The Electronic Signature and the Problems of Its Practical Applicable. International Journal of Humanities and Social Science, 6(12), 67-72.

Assuresign. (2018, March 28). Defining E-Signature: Sound, Symbol or Process. Retrieved from https://www.assuresign.com/sound-symbol-or-process/

Bagherian, A., Nasaband, Y. E., Heidari, H., \& Ebrahimi, M. (2014). The Role of Electronic Signature in the Third Millennium of the Development of International Trade Transactions. International Journal of Information Systems and Social Change, 5(2), 39-52. doi:10.4018/ijissc.2014040105

Bain, M., \& Subirana, B. (2003). E-commerce oriented software agents. Computer Law \& Security Review, 19(3), 201-211. doi:10.1016/s0267-3649(03)00303-0

Ghosh, A., Mahdian, M., McAfee, R. P., \& Vassilvitskii, S. (2015). To Match or Not to Match. $A C M$ Transactions on Economics and Computation, 3(2), 1-18. doi:10.1145/2745801

Government of Jordan. (2001). Electronic Transactions Law No. (85) of 2001. Electronic Transaction Law.

Hanadi, D. (2015). The contract Electronic. (Unpublished PHD dissertation). University of Azhari Leader.

Ihab, A., Ismail, S. M., \& Kamat, V. R. (2006). Evaluation of Legal Risks for E-Commerce in Construction. Journal of Professional Issues in Engineering Education and Practice, 132(4).

Justia. (2018). O'Connor v. Uber, No. 14-16078 (9th Cir. 2018). Retrieved from https:/law.justia.com/cases/federal/appellate-courts/ca9/14-16078/14-16078-2018-09-25.html

Kalloniatis, C., Mouratidis, H., Vassilis, M., Islam, S., Gritzalis, S., \& Kavakli, E. (2014). Towards the design of secure and privacy-oriented information systems in the cloud: Identifying the major concepts. Computer Standards \& Interfaces, 36(4), 759-775. doi:10.1016/j.csi.2013.12.010

Lexology. (2011, April 13). Barwick v. GEICO. Retrieved from https://www.lexology.com/library/detail.aspx?g=2bd1dba7-a283-4c24-8819-f492075611f5

McKendrick, E. (2014). Contract law: text, cases, and materials. Oxford University Press (UK).

Moringiello, J. M., \& Reynolds, W. L. (2005). Survey of the Law of Cyberspace: Electronic Contracting: Cases 2004-2005. Business Lawyer, 61, 433.

Mormann, M. D. (2015). DPH Consolidated Contract Addendum.

Mukherjee, A., \& Nath, P. (2003). A model of trust in online relationship banking. International Journal of Bank Marketing, 21(1), 5-15. doi:10.1108/02652320310457767

Ramokanate, L. L. (2014). A legal framework for the treatment of input errors in electronic contracts (Doctoral dissertation). North-West University.

Shnikat, M., Alzubi, A., Aljaber, M., \& Alnsoor, A. (2017). The legal framework of electronic contract in the Jordanian legislation. Global Journal of Politics and Law Research, 5(5), 46-62.

Statista. (2019). eCommerce - worldwide Market Forecast. Retrieved from https://www.statista.com/outlook/243/100/ecommerce/worldwide.

United Nations. (2001). UNCITRAL Model Law on Electronic Signatures with Guide to Enactment 2001. UNCITRAL

Whitley, E. A., Gal, U., \& Kjaergaard, A. (2014). Who do you think you are? A review of the complex interplay between information systems, identification and identity. European Journal of Information Systems, 23(1), 17-35. doi:10.1057/ejis.2013.34

\section{List of Abbreviations}

ECD 99/93: DIRECTIVE 1999/93/ EC of the European Parliament and of the Council of 13 December 1999 on a Community framework for electronic signatures

E-SIGN- Electronic Signatures in Global and National Commerce Act

E-Signature- Electronic Signature

ETL: Electronic Transaction Law

ICT: Information and Communications Technology

UNCITRAL: The United Nations Commission on International Trade Law

USD: United States Dollar 